\title{
EDITORIAL
}

\section{Emociones en la educación en Colombia, algunas reflexiones}

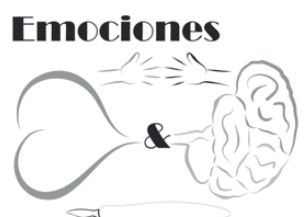

Educación

Sin lugar a dudas, es un privilegio presentar el volumen 10 número 24 de la Revista Praxis \& Saber -Emociones y educación-, no solo por celebrar el impacto creciente de la revista; por el incremento de las investigaciones y hallazgos en educación, pedagogía y didáctica; por los encuentros y vínculos que se realizarán a propósito del $V$ Congreso Internacional de Investigación y Pedagogía, Escuela, Maestro y Estudio, perspectivas contemporáneas; por la pertinencia e importancia social y educativa de la Maestría en Educación de la Universidad Pedagógica y Tecnológica de Colombia [UPTC]; sino porque este momento es significativo, debido a que tuvieron que transcurrir muchos años, momentos y experiencias para que en la Facultad de Ciencias de la Educación - y en gran medida en la UPTC- se configurara el momento para poder hablar sin reservas y con soporte académico e investigativo de las emociones y su relación con la educación.

Desde luego, la línea de Emociones \& Educación del grupo de investigación Cacaenta y su permanente trabajo conjunto con el Grupo de Investigación de la Junta de Andalucía [Dedica] "Desarrollo, educación, diversidad y cultura: análisis interdisciplinar" (HUM-742) de la Universidad de Granada, España, ha sido protagonista de este proceso, el cual ha sido potenciado por 
la apertura, flexibilidad y perspectiva de la Maestría en Educación de la UPTC, la cual permitió que esta línea del grupo se convirtiera también en una de las líneas de investigación del programa.

En cualquier caso, el estudio de las emociones tiene gran relevancia en el contexto global en diferentes áreas, pero además las indagaciones en el ámbito de la educación son fundamentales. Día a día se presentan hallazgos que permiten nuevas perspectivas y cambios orientados al fortalecimiento de las personas, al aprendizaje y al éxito en la vida, así como a las relaciones y vínculos de estudiantes, profesores, padres de familia y demás actores del contexto educativo.

En esta dirección es importante recordar que las emociones han sido abordadas a lo largo de la historia por distintas disciplinas como la biología, la filosofía -desde los griegos-, el psicoanálisis -en especial freudiano-, la psicología - conductista, cognitiva y positiva-, la sociología, el análisis facial de las emociones, la neurociencia afectiva y la educación. Aun así, por mucho tiempo las emociones fueron consideradas como irrelevantes y accesorias, motivo por el cual había que ignorarlas, e incluso reprimirlas. Desde el contexto escolar la tendencia fue la misma, es decir, se creía que había que excluirlas porque la escuela estaba encargada del pensamiento y el aprendizaje. Se pensaba que las emociones no tenían nada que ver con los procesos cognitivos (Buitrago \& Herrera, 2013), motivo por el cual era impertinente permitir su presencia en las aulas.

Desde luego, y por fortuna, esta perspectiva se ha modificado con los años, en primer lugar, por el creciente incremento de resultados de investigación, los cuales aumentaron desde que en 1990 se publicó un artículo académico de Peter Salovey y John Mayer, en el que se desarrolló el concepto de inteligencia emocional [IE], lo cual sirvió de base para el fortalecimiento del constructo (Buitrago, 2012); pero también, desde la perspectiva del público en general, cuando en 1995 Daniel Goleman publica su best seller, Inteligencia Emocional.

Ahora bien, no se deben dejar de mencionar los relevantes aportes en el estudio de las emociones de algunos investigadores, como Candace Pert, quien en 1973 descubrió el receptor opiáceo, 
encargado de recibir la información emocional segregada por el cerebro y transportada por los neurotrasmisores denominados neuropéptidos (Pert, 1999). Paul Ekman es pionero en el estudio de las emociones universales (Ekman, 1994, 1999, 2003), y dedica gran parte de sus investigaciones a las expresiones y microexpresiones faciales y su reconocimiento (Ekman, 1993, 2015). De igual manera, Richard Davidson desde la perspectiva de la neurociencia ha planteado propuestas como la teoría del perfil emocional del cerebro (Davidson \& Begley, 2012) y ha llevado el estudio de las emociones a niveles de gran complejidad (Davidson, 2001, 2003a, 2003b; Davidson \& Irwin, 1999; Davidson, Jackson \& Kalin, 2000; Davidson, Shackman \& Maxwell, 2004; Kesebir, Gasiorowska, Goldman, Hirshberg \& Davidson, 2019), por lo que se le conoce como el padre de la neurociencia afectiva.

Desde el lugar de la educación, son múltiples los estudios y programas de aprendizaje social y emocional SEL - Social and Emotional Learning - que se han diseñado e implementado con éxito en el contexto global (Agulló, Filella, Soldevila \& Ribes, 2011; Brackett, Mayer \& Warner, 2004; Cherniss, Extein, Goleman \& Weissberg, 2006; Eren, Ergun \& Altintas, 2009; Gil-Olarte, Palomera \& Brackett, 2006; Greenberg et al., 2003), aunque se requiere enfatizar que la mayoría se han implementado en Estados Unidos de América (Clouder et al., 2008). En esta dirección, el programa Promoting Alternative Thinking Strategies [PATHS], por ejemplo, trabaja en el bienestar de los niños a partir de algunos sentimientos básicos como la felicidad, la tristeza, el miedo y la seguridad, para ir abordando otros más complejos de manera progresiva como el orgullo o la decepción, para pasar luego a la humillación, la vergüenza y el rechazo (Greenberg, 2003).

El programa PATHS, en español Pensamiento, afecto y trabajo de habilidades sociales, se originó en la Universidad de Pensilvania y se ha implementado además de los Estados Unidos de América, en otros países de Europa y América, entre ellos Inglaterra, Australia, Holanda, Bélgica, Alemania, México, Colombia, Argentina, Venezuela y Chile. Los estudios que han analizado el impacto del programa PATHS han encontrado una incidencia positiva en cuanto a la reducción de la agresividad, la mejora de los vínculos y procesos de socialización y el desarrollo de habilidades emocionales (Kam, 
Greenberg \& Kusche, 2004; Kam, Greenberg \& Walls, 2003; Riggs, Greenberg, Kusche \& Pentz, 2006), incluidos los niños de preescolar (Domitrovich, Cortes, \& Greenberg, 2007).

A pesar del importante volumen de investigaciones en el ámbito global orientadas al estudio de las emociones, y en particular al de la IE y su relación, impacto e incidencia en el contexto escolar, lo cierto es que los estudios en este sentido en Latinoamérica aún son reducidos, en particular en Colombia (Buitrago, 2012), aunque también hay un importante número de publicaciones científicas creciente en la actualidad. Además, pese a la experiencia de la implementación de algunos programas como PATHS y de algunas otras experiencias particulares relacionadas con las emociones y la IE, hasta hace pocos años el Ministerio de Educación Nacional de Colombia [MEN] empezó a reconocer la evidencia empírica respecto a la importancia de las emociones en la escuela y en los procesos que en ella se desarrollan, y empezó a señalar la necesidad de que el sistema educativo colombiano empezara a vincular las emociones de manera formal en sus procesos, reflexiones, normas y políticas. Es por ello que dentro de las políticas orientadas a fortalecer la calidad educativa de la educación preescolar e infantil, a través de la Comisión Intersectorial para la Atención Integral de la Primera Infancia [CIPI] se propone una plataforma digital para difundir material respecto a las competencias socioemocionales. Asimismo, se pretende consolidar el sistema de convivencia escolar en la educación básica y media, soportado en el fortalecimiento de las competencias transversales y socioemocionales, al integrarlas a las competencias ciudadanas y al índice sintético de calidad educativa (MEN, 2019).

Esta perspectiva y apuesta, sin lugar a dudas, es relevante y establece un horizonte de esperanza en términos de una mejora sustancial de la escuela colombiana y de un sistema que centre sus objetivos de manera contundente en el desarrollo y bienestar del alumnado y del profesorado en primer lugar, pero también de los demás actores del contexto educativo para potenciar los procesos de aprendizaje y pensamiento. Sin embargo, y quizá de manera inevitable, siguen siendo los organismos internacionales de control económico los que asumen el protagonismo de estos procesos (Buitrago, 2016, 2018) y como es lógico, terminan estableciendo los lineamientos, directivas y sesgos respectivos. 
Desde esta óptica, se puede señalar, por ejemplo, que en 2015 un equipo de la OCDE visitó Bogotá para socializar un estudio longitudinal orientado a medir las habilidades socioemocionales de estudiantes de varias ciudades del mundo, incluida Bogotá. Dicho estudio pretende abordar aspectos de la familia, la escuela y el contexto, y culmina en 2020. En esta misma dirección, en 2017, el Departamento Nacional de Planeación, el MEN y la oficina de Bogotá del Banco Mundial adaptaron para Colombia el programa propuesto por el Banco Mundial - Paso a paso, estrategia de formación de competencias socioemocionales en la educación secundaria y media-.

$\mathrm{Al}$ respecto, según Raadschelders (2019), se debe advertir que también se hace presente el abuso manipulador de la democracia y que las emociones también cuentan con un importante potencial para la manipulación, en muchas ocasiones, en las situaciones sociales (Meng, 2018). En cualquier caso, lo cierto es que el pensamiento pedagógico y las políticas educativas no deberían seguir en manos de quienes propenden por un horizonte neoliberal, en el que el desarrollo y bienestar humano pasa a un segundo plano para que el desarrollo y la sostenibilidad económica se conviertan en objetivo primordial, mientras se privilegia el enriquecimiento y la manipulación económica para la consecución de poder.

Dejando de lado esta perspectiva, la cual no se puede perder de vista, es importante establecer que tanto el modelo de habilidades emocionales de Salovey \& Mayer (Brackett \& Salovey, 2006; Fernández-Berrocal \& Extremera, 2006; Mayer \& Salovey, 1997, 2009; Mayer, Salovey \& Caruso, 2000, 2002, 2008; Mayer, Salovey, Caruso \& Sitarenios, 2001), así como el socioemocional de Bar-On (Bar-On, 1997, 2006; Fernández-Berrocal \& Extremera, 2006) tienen gran relevancia para el contexto educativo. El primero establece cuatro habilidades fundamentales: a) la percepción, valoración y expresión de la emoción, b) la facilitación emocional del pensamiento, c) la comprensión y análisis de las emociones haciendo uso del conocimiento emocional y d) la regulación reflexiva de las emociones para promover el crecimiento emocional e intelectual.

Desde el modelo socioemocional se hace referencia al ámbito intrapersonal, al interpersonal, a la adaptabilidad y al manejo del 
estrés, aspectos que configuran en este modelo el nivel de coeficiente emocional, el cual también se ve afectado por el estado general de ánimo y la impresión positiva que tienen las personas de sí mismas. En este sentido, al pensar en los alumnos, los lineamientos establecidos en dichos modelos permiten un proceso de desarrollo emocional desde los primeros niveles educativos, hasta los más complejos en el nivel superior.

Estos modelos permiten abordar aspectos como el reconocimiento de las emociones propias y de los demás, la expresión adecuada de los sentimientos y las emociones, el fortalecimiento de la empatía afectiva y cognitiva, así como el control de los impulsos a través de la gestión de las emociones; aspectos que se relacionan con el desarrollo de la individualidad y el reconocimiento de las diferencias interindividuales, la autoestima y la seguridad interior, y paralelo a ello, con la configuración de vínculos y relaciones positivas con las demás personas (Fernández-Berrocal \& Extremera, 2002; Herrera, Buitrago-Bonilla \& Cepero, 2017; Herrera, Buitrago, Lorenzo \& Badea, 2015).

Desde la óptica del profesorado, no solo es importante su propio desarrollo personal y profesional, sino también el cultivo del amor por el conocimiento, la pasión de la enseñanza, la comunicación asertiva, el fomento de la creatividad, el abordaje de la motivación intrínseca y extrínseca, la búsqueda de las habilidades y talentos en los niños, y la libertad para que cada alumno pueda encontrar su camino y configurar su propia vida, entre otras (Herrera \& Buitrago, 2014; Robinson, 2015).

En este momento, se hace relevante enfatizar en la importancia de los programas de formación del profesorado (Buitrago, Ávila \& Cárdenas, 2017; Buitrago-Bonilla \& Cárdenas-Soler, 2017; Herrera, Buitrago \& Ávila, 2016; Mariño, Pulido \& Morales, 2016; Martín-Gutiérrez, Conde-Jiménez \& Mayor-Ruiz, 2014; Palomera, Fernández-Berrocal \& Bracket, 2008; Pulido \& Gómez, 2017), para Colombia, ofertados por las normales superiores, en particular por los programas de formación complementaria [PFC] y por las facultades de educación a través de los programas de licenciatura, ya que durante estos procesos el profesorado tiene la posibilidad de integrar a sus pensamientos y prácticas una mirada amplia, 
flexible y creativa, pero estructurada y crítica, en donde el desarrollo de habilidades emocionales se establece no solo como posible, sino como fundamental para el posterior ejercicio profesional y el desarrollo integral de los alumnos.

Por último, es importante explicitar el protagonismo de las emociones para el aprendizaje, el conocimiento y el pensamiento, ya que ellas los dificultan, entorpecen, facilitan o potencian, es decir, se puede enfatizar en que la configuración emocional y el estado interior de las personas operan como moduladores y reguladores de los pensamientos, aspecto que incide en el aprendizaje, el rendimiento académico y la felicidad, en síntesis, en la manera de estructurar, configurar y, ante todo, utilizar el pensamiento.

Queridos lectores, en este número de Praxis \& Saber podrán disfrutar del documento "Educación emocional para el desarrollo de competencias emocionales en niños y adolescentes", donde la Dra. Núria Pérez-Escoda y la Dra. Gemma Filella Guiu presentan una propuesta orientada al desarrollo e implementación de programas de desarrollo de competencias emocionales para el contexto escolar. En la misma dirección y en compañía de la Dra. Ruth Nayibe Cárdenas Soler compartimos el artículo "Coeficiente emocional en niños y adolescentes de Boyacá, Colombia. Estudio comparativo" el cual plantea un análisis respecto a la IE de niños y jóvenes estudiantes de Colombia.

Así mismo, el Dr. Natalio Extremera, el magister Sergio MéridaLópez, el Dr. Nicolás Sánchez-Álvarez, la Dra. Cirenia Quintana-Orts y la Dra. Lourdes Rey exponen los resultados de una investigación realizada con docentes españoles de primaria y secundaria que analizó la relación entre inteligencia emocional y engagement apoyo social organizacional de compañeros y supervisores-, en su texto "Un amigo es un tesoro: inteligencia emocional, apoyo social organizacional y engagement docente". De igual manera, la Dra. Raquel Palomera, la Dra. Elena Brioñez Pérez y la Dra. Alicia Gómez Linares exponen los resultados de un proyecto pionero desarrollado en la Universidad de Cantabria que se orienta a fortalecer la formación en valores y competencias personales de los estudiantes de magisterio, en el artículo "Formación en valores y competencias socioemocionales para docentes tras una década de innovación". 
Sumado a ello, la Dra. Amelia Barrientos Fernández, la Dra. Amaya Arigita García y el Dr. Roberto Sánchez Cabrero en su colaboración "Formación emocional del profesorado y gestión del clima de su aula" analizan la relación entre la capacidad para gestionar el clima socioemocional del aula y la formación emocional del profesorado. En el artículo "Comunicación asertiva en profesores: diagnóstico y propuesta educativa”, la magíster Dora Cristina Cañas Betancur y la magíster Jacqueline Hernández Sánchez presentan un diagnóstico respecto a la comunicación asertiva y su vínculo con la configuración sociodemográfica de una institución educativa pública.

En el mismo sentido, el artículo "Las habilidades socioemocionales en los docentes, herramientas de paz en la escuela" analiza la incidencia de las habilidades socioemocionales de los docentes en los ambientes de paz en la escuela, presentado por la joven investigadora Adriana Marien Gutiérrez-Torres y la estudiante de maestría Sandra Jackeline Buitrago-Velandia. Por su parte, el magíster John Fredy Henao Arias y el magister Andrés Elías Marín Rodríguez plantean un análisis de la mediación de las emociones en los procesos comunicativos de profesores y estudiantes, así como en la toma de decisiones en instituciones educativas del departamento de Caldas, en el texto "El proceso de enseñanza desde el prisma de las emociones docentes".

La Dra. Laura Sánchez Calleja, en su escrito "La formación inicial docente en una universidad chilena: estudiando el ámbito emocional", analiza la manera en que se hace el abordaje emocional en la Universidad Metropolitana de Ciencias de la Educación de Santiago de Chile en la formación inicial del profesorado. En la misma dirección, el artículo "Competencias socioemocionales de maestros en formación y egresados de programas de educación”, de la Dra. María Alexandra Rendón Uribe, evalúa las competencias emocionales de maestros en formación y egresados de la Facultad de Educación de la Universidad de Antioquia.

Continuando, el artículo "Claroscuros de la inteligencia emocional iluminan la utopía”, del Dr. Luis Rodolfo Ibarra Rivas, aborda criterios referentes a la alfabetización emocional y se centra en aspectos como la emoción, el sentimiento y el afecto, todo ello para hacer énfasis en el humanismo y el altruismo. Sumado a lo anterior, el doctorando Felipe Nicolás Mujica Johnson y la Dra. Nelly del Carmen Orellana Arduiz 
en su escrito "Emociones en educación física desde la perspectiva constructivista: análisis del currículo de España y Chile" propone un análisis en pro de una educación integral, desde la perspectiva de la educación física, de los currículos de España en Chile.

En el mismo sentido, el magíster Jhon Carlos Cortés Murillo y la magíster Ita del Pilar Perea Baena se enfocan en la perspectiva de tres maestros respecto a estudiantes que se encuentran en gestación. Para ello, analizan el sentir-pensar-actuar de ellos en el artículo "Gestantes adolescentes en la perspectiva de tres docentes de educación física escolar". Por otro lado, se analizan los resultados de algunos estudiantes que presentaron las Pruebas Saber 11 y más adelante las pruebas Saber Pro, para hacer un análisis al respecto, en el documento "La educación secundaria y superior en Colombia vista desde las pruebas Saber", de la matemática Mayerlin Castro y el Dr. Jorge Ruiz.

También se presenta el artículo "The influence of coordination on the attention stability of children with different nervous system typologies" del Dr. Georgiy Georgievich Polevoy, en el que se presenta un experimento pedagógico que analizó la relación de las habilidades de coordinación en el movimiento con la estabilidad en la atención de los alumnos. Esta edición de Praxis \& Saber cierra con la traducción del texto "La escuela como práctica y tecnología de la pertenencia al mundo" de Jan Masschelein, a proposito del libro Defensa de la Escuela. Una Cuestión Pública, realizada por el Lic. Brayan Andrés Morantes Martinez y el Dr. Juan Guillermo Díaz Bernal. Esperamos que disfruten, cuestionen y se emocionen con la lectura de estas aportaciones.

Dra. Lucía Herrera Torres Universidad de Granada, España. Grupo de Investigación de la Junta de Andalucía "Desarrollo, Educación, Diversidad y Cultura: Análisis interdisciplinar" (HUM-742) Editora Invitada

Dr. Rafael Enrique Buitrago Bonilla

Universidad Pedagógica y Tecnológica de Colombia. Grupos de Investigación Cacaenta y DEDICA. Línea de Investigación Emociones \& Educación.

Editor Invitado 


\section{Referencias}

Agulló, M., Filella, G., Soldevila, A., \& Ribes, R. (2011). Evaluación de la educación emocional en el ciclo médio de Educación Primaria. Revista de Educación, 354, 765-783.

Bar-On, R. (1997). Bar-On Emotional Quotient Inventory: Technical Manual. Toronto: Multi Health System Inc.

Bar-On, R. (2006). The Bar-On model of Emotional-Social Intelligence (ESI). Psicothema, 18(Suppl.), 13-25.

Brackett, M., \& Salovey, P. (2006). Measuring emotional intelligence with the Mayer-Salovery-Caruso Emotional Intelligence Test (MSCEIT). Psicothema, 18(Suppl.), 34-41.

Brackett, M., Mayer, J., \& Warner, R. (2004). Emotional intelligence and its relation to everyday behavior. Personality and Individual Differences, 36, 1387-1402. http://dx.doi.org/10.1016/So191-8869(03)oo236-8

Buitrago, R., Ávila, A., \& Cárdenas, R. (2017). El sentido y el significado atribuido a las emociones por el profesorado en formación de la Universidad Pedagógica y Tecnológica de Colombia. Contextos Educativos, 20, 77-93. http://doi.org/10.18172/con.2998

Buitrago, R. (2012). Contexto Escolar e Inteligencia Emocional en Instituciones Educativas Públicas del Ámbito Rural y Urbano del Departamento de Boyacá (Colombia) (Tesis doctoral, Universidad de Granada, Granda, España). Recuperado de http://hera.ugr.es/ tesisugr/20956575.pdf

Buitrago, R. (2016). La formación de maestros como alternativa para una educación de calidad. Praxis \& Saber, 7(15), 9-16. http://dx.doi. org/10.19053/22160159.v7.n15.2016.5720

Buitrago, R. (2018). Transformaciones sociales y educativas desde procesos investigativos. Praxis \& Saber, 9(21), 9-20. https://doi. org/10.19053/22160159.v9.n21.2018.8922

Buitrago, R., \& Herrera, L. (2013). Matricular las emociones en la escuela, una necesidad educativa y social. Praxis \& Saber, 8(4), 87-108.

Buitrago-Bonilla, R., \& Cárdenas-Soler, R. (2017). Emociones e identidad profesional docente: relaciones e incidencia. Praxis \& Saber, 8(17), 225-247. https://doi.org/10.19053/22160159.v8.n17.2018.7208

Cherniss, C., Extein, M., Goleman, D., \& Weissberg, R. (2006). Emotional Intelligence: What Does the Research Really Indicate? Educational Psychologist, 41(4), 239-245. https://doi.org/10.1207/ S15326985ep4104_4 
Clouder, C., Dahlin, B., Diekstra, R., Fernández-Berrocal, P., Heys, B., Lantieri, L., \& Paschen, H. (2008). Educación Emocional y Social, Análisis Internacional. España: Informe Fundación Marcelino Botín.

Davidson, R. (2001). Toward a biology of personality and emotion. Annual National Yearbook of Academy of Sciences, 935, 191-207. https://doi. org/10.1111/j.1749-6632.2001.tbo3481.x

Davidson, R. (2003a). La neurociencia de la emoción. En D. Goleman (Ed.), Emociones Destructivas, cómo entenderlas y superarlas (pp. 104118). Barcelona: Editorial Kairós.

Davidson, R. (2003b). Seven sins in the study of emotion: Correctives from affective neuroscience. Brain and Cognition, 52, 129-132. https://doi. org/10.1016/So278-2626(03)00015-0

Davidson, R., \& Begley, S. (2012). El perfil emocional de tu cerebro. Barcelona: Ediciones Destino.

Davidson, R., \& Irwin, W. (1999). The functional neuroanatomy of emotion and affective style. Trends in Cognitive Sciences, 3(1), 11-21. http:// dx.doi.org/10.1016/S1364-6613(98)01265-0

Davidson, R., Jackson, D., \& Kalin, N. (2000). Emotions, plasticity, context and regulation: perspectives from affective neuroscience. Psychological Bulletin, 126(6), 890-906. http://dx.doi.org/10.1037/00332909.126.6.890

Davidson, R., Shackman, A., \& Maxwell, J. (2004). Asymmetries in face and brain related to emotion. TRENDS in Cognitive Sciences, 8, 389-391. http://dx.doi.org/10.1016/j.tics.2004.07.006

Domitrovich, C., Cortes, R., \& Greenberg, M. (2007). Improving young children's social and emotional competence: A randomized trial of the preschool "PATHS" curriculum. Journal of Primary Prevention, 28(2), 67-91. http://dx.doi.org/10.1007/s10935-007-0081-0

Ekman, P. (1993). Facial Expression and Emotion. American Psychologist, 48(4), 384-392.

Ekman, P. (1994). Strong evidence for universals in facial expresions: A reply to Russell's mistaken critique. Psychological Bulletin, 115, 268-287.

Ekman, P. (1999). Basic Emotions. En T. Dalgleish, \& M. Power (Eds.), Handbook of Cognition and Emotion (pp. 45-60). Sussex, U.K.: John Wiley \& Sons, Ltd.

Ekman, P. (2003). La universalidad de las emociones. En D. Goleman (Ed.), Emociones Destructivas, cómo entenderlas y superarlas (pp. 71-93). Barcelona: Editorial Kairós. 
Ekman, P. (2015). El rostro de las emociones. Barcelona: RBA libros S.A.

Eren, E., Ergun, E., \& Altintas, O. (2009). The Relationship Between School Success and the Emotional Intelligence of Primary School Headmasters and Teachers. Journal of American Academy of Business, Cambridge, 15(1), 224-228.

Fernández-Berrocal, P., \& Extremera, N. (2002). La Inteligencia Emocional como una habilidad esencial en la Escuela. Revista Iberoamericana de Educación, 29, 1-6. Recuperado de http://www.rieoei.org/ deloslectores/326Berrocal.pdf

Fernández-Berrocal, P., \& Extremera, N. (2006). Emotional intelligence: A theoretical and empirical review of its first 15 years of history. Psicothema, 18(Suppl.), 7-12.

Gil-Olarte, M., Palomera, R., \& Brackett, M. (2006). Relating emotional intelligence to social competence and academic achievement in high school students. Psicothema, 18(Suppl.), 118-123.

Goleman, D. (1995). Emotional Intelligence. New York: Bantam Books.

Greenberg, M. (2003). La educación del corazón. En D. Goleman (Ed.), Emociones Destructivas, cómo entenderlas y superarlas (pp. 146158). Barcelona: Editorial Kairós.

Greenberg, M., Weissberg, R., O’Brien, M., Zins, J., Fredericks, L., Resnik, H., \& Elias, M. (2003). Enhancing school-based prevention and youth development through coordinated social and emotional learning. American Psychologist, 58, 466-474. http://dx.doi. org/10.1037/0003-066X.58.6-7.466

Herrera, L., \& Buitrago, R. (2014). Emociones, inteligencia emocional, educación y profesorado. En L. Herrera (Coord.), Retos y desafíos actuales de la Educación Superior desde la perspectiva del profesorado universitario (pp. 179-203). Madrid: Síntesis.

Herrera, L., Buitrago, R., \& Ávila, A. (2016). Empathy in future teachers of the Pedagogical and Technological University of Colombia. New Approaches in Educational Research, 5(1), 30-37. https://doi. org/10.7821/naer.2016.1.136

Herrera, L., Buitrago, R., Lorenzo, O., \& Badea, M. (2015). Socio-Emotional Intelligence in Colombian Children of Primary Education. An analysis in rural and urban settings. Procedia - Social and Behavioral Sciences, 203, 4-10. https://doi.org/10.1016/j.sbspro.2015.08.251

Herrera, L., Buitrago-Bonilla, R., \& Cepero S. (2017). Emotional Intelligence in Colombian Primary School Children. Location and Gender. Universitas Psychologica, 16(3), 1-10. https://doi.org/10.11144/ Javeriana.upsy16-3.eips 
Kam, C., Greenberg, M., \& Kusche, C. (2004). Sustained effects of the PATHS curriculum on the social and psychological adjustment of children in special education. Journal of Emotional and Behavioral Disorders, 12(2), 66-78. http://dx.doi.org/10.1177/10634266040120020101

Kam, C., Greenberg, M., \& Walls, C. (2003). Examining the role of implementation quality in school-based prevention using the PATHS curriculum. Prevention Science 4(1), 55-63. http://dx.doi. org/10.1023/A:1021786811186

Kesebir, P., Gasiorowska, A., Goldman, R., Hirshberg, M., \& Davidson, R. (2019). Emotional Style Questionnaire: A multidimensional measure of healthy emotionality. Psychological Assessment, Advance online publication. http://dx.doi.org/10.1037/pasoooo745

Mariño, L., Pulido, Ó., \& Morales, L. (2016). Actitud Filosófica, Infancia y Formación de Maestros. Praxis \& Saber, 15(7), 81-101. http://dx.doi. org/10.19053/22160159.v7.n15.2016.5724

Martín-Gutiérrez, A., Conde-Jiménez, J., \& Mayor-Ruíz, C. (2014). La identidad profesional docente del profesorado novel universitario. REDU, Revista de Docencia Universitaria, 12(4), 141-160.

Mayer, J., \& Salovey P. (1997). What is emotional intelligence? En P. Salovey, \& D. Sluyter (Eds.), Emotional development and emotional intelligence: implications for educators (pp. 3-34). New York: Basic Books.

Mayer, J., \& Salovey P. (2009). ¿Qué es inteligencia emocional? En J. Mestre, \& P. Fernández-Berrocal (Coords.), Manual de inteligencia emocional (pp. 25-45). Madrid: Ediciones Pirámide.

Mayer, J., Salovey, P., \& Caruso, D. (2000). Models of emotional intelligence. En R. Sternberg (Ed.), Handbook of Intelligence (pp. 396-420). New York: Cambridge.

Mayer, J., Salovey, P., \& Caruso, D. (2002). Mayer-Salovey-Caruso Emotional Intelligence Test (MSCEIT). Version 2.o. Toronto, Canadá: Multi-Health Systems.

Mayer, J., Salovey, P., \& Caruso, D. (2008). Emotional Intelligence, New Ability or Eclectic Traits?. American Psychologist, 63(6), 503-517. doi: 10.1037/0003-066X.63.6.503

Mayer, J., Salovey, P., Caruso, D., \& Sitarenios, G. (2001). Emotional intelligence as a standard intelligence. Emotion, 1, 232-242. doi: 10.1037/1528-3542.1.3.232

Meng, Q. (2018). Intercultural Communication Strategies in Diplomatic Relations: A Case Study of Donald Trump's First Visit to China. 
Cross-Cultural Communication, 14(4), 74-82. http://dx.doi. org/10.3968/10684

Ministerio de Educación Nacional. (2019). Plan Estratégico Institucional 2019-2022, Educación de calidad para un futuro con oportunidades para todos. Bogotá: Ministerio de Educación Nacional.

Palomera, R., Fernández-Berrocal, P., \& Bracket, M. (2008). La inteligencia emocional como una competencia básica en la formación inicial de los docentes: algunas evidencias. Revista electrónica de investigación psicoeducativa, 15(6), 437-454.

Pert, C. (1999). Molecules of Emotion: The Science Behind Mind-Body Medicine. New York: Touchstone.

Pulido, Ó., \& Gómez, L. (2017). Del enseñar y el aprender. Praxis \& Saber, 8(18), 9-14. https://doi.org/10.19053/22160159.v8.n17.2018.7220

Raadschelders, J. (2019). Challenges of Governing Democratic Regimes: Public Administration as Counterweight to Populism, Partisanship, and Rent-Seeking. Manuscrito sin publicar. Washington D.C.: The Ohio State University.

Riggs, N., Greenberg, M., Kusche, C., \& Pentz, M. (2006). The mediational role of neurocognition in the behavioral outcomes of a social-emotional prevention program in elementary school students: effects of the PATHS curriculum. Prevention Science, 7, 91-102. http://dx.doi.org/10.1007/ S11121-005-0022-1

Robinson, K. (2015). How Creativity, Education and the Arts Shape a Modern Economy. Arts and Minds, Conversations about the Arts in Education. Recuperado de https://www.ecs.org/clearinghouse/6o/51/6051.pdf

Salovey, P., \& Mayer, J. (1990). Emotional Intelligence. Imagination, Cognition and Personality, 9, 185-211. 